\title{
Comparison of Peak Aerobic Capacity Between the Treadmill and a Skiing Ergometer
}

\author{
Original Research
}

Hannah E. Nelson ${ }^{1}$, Andy M. Bosak ${ }^{2}$, Russell K. Lowell ${ }^{3}$, Maggie J. McDermott ${ }^{2}$, Branden M. Ziebell ${ }^{2}$, Moroni A. de Moors $^{2}$, Anna C. Blackley ${ }^{4}$, Samuel J. Arter ${ }^{2}$

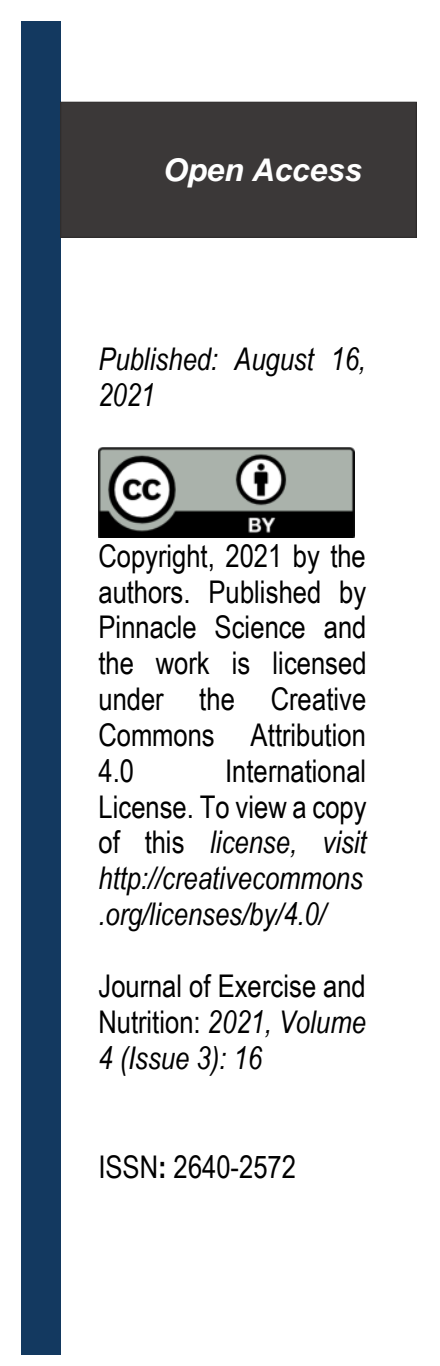

\author{
${ }^{1}$ Health, Exercise Science, and Recreation Management, University of Mississippi, \\ Mississippi/USA \\ ${ }^{2}$ Allied Health Professions, Liberty University, Virginia/USA \\ ${ }^{3}$ Kinesiology, Mississippi State University, Mississippi/USA \\ ${ }^{4}$ Physical Therapy, Western Carolina University, North Carolina / USA
}

\begin{abstract}
Introduction: The Concept2 SkiErg is increasing in popularity and is widely used to provide a low impact total body workout. Because of these benefits, the SkiErg could be an ideal tool for fitness testing. Therefore, the purpose of this study was to compare $\mathrm{VO}_{2}$ peak values elicited from a treadmill (TM) and SkiErg (SE) graded exercise test (GXT).

Methods: Twenty-two females completed two GXT protocols to volitional exhaustion on a TM and SE. Peak $\mathrm{VO}_{2}, \mathrm{HR}, \mathrm{VE}, \mathrm{TTE}$, and RER were compared using paired-samples t-tests with significant differences at $\mathrm{p} \leq 0.05$. Max $R P E$ was compared using a Wilcoxon Signed Rank Test.

Results: TM was significantly greater than SE for $\mathrm{VO}_{2 \max }(43.82 \pm 1.07$ vs $33.97 \pm 5.01 \mathrm{ml} / \mathrm{kg} / \mathrm{min}, \mathrm{p}<0.01)$, HR (189 \pm 8 vs $182 \pm 11 \mathrm{bpm}, \mathrm{p}<0.01), \mathrm{RPE}$ $(18.91 \pm 1.11 \mathrm{vs} 17.26 \pm 2.03, \mathrm{p}=0.02), \mathrm{VE}(95.44 \pm 11.26$ vs $86.21 \pm 2.90 \mathrm{~L} / \mathrm{min}$, $\mathrm{p}=0.015)$, and TTE (550.16 \pm 137.56 vs $391.86 \pm 81.20$ sec, $\mathrm{p}<0.01)$. RER was significantly greater on the SE $(1.16 \pm 0.08$ vs $1.12 \pm 0.06, \mathrm{p}=0.024)$.

Conclusion: Results suggest that TM elicits higher max values for $\mathrm{VO}_{2}, \mathrm{HR}$, TTE, VE, and RPE compared to SE, while SE elicits a higher RER in the current population. SE could be used as an alternative mode of testing in averagely-fit females but does not directly compare to TM values.
\end{abstract}

Key Words: Oxygen consumption, protocol, SkiErg

Corresponding author: Hannah Nelson, hnelson2@olemiss.edu

\section{Introduction}

Maximal oxygen uptake $\left(\mathrm{VO}_{2 \mathrm{max}}\right)$ is considered the gold standard measurement for assessing an individual's cardiorespiratory fitness in both clinical and athletic populations ${ }^{1,2}$. $\mathrm{VO}_{2 \max }$ refers to how much oxygen is taken in and used by the body and relies heavily on how well the heart can accommodate the need for oxygen throughout the rest of the body ${ }^{2}$. $\mathrm{VO}_{2 \max }$ is determined through either direct measurement or indirect estimation using graded exercise tests designed for aerobic capacity ${ }^{1}$. Treadmill (TM) and cycle ergometers are the most common modalities utilized when assessing an individual's maximal aerobic capacity ${ }^{3-6}$. However, additional protocols have been developed on alternative modalities to provide testing to accommodate a variety of populations. Such alternative protocols range from arm- 
crank ergometry ${ }^{7,8}$, Digi-Jump machine ${ }^{9}$, Elliptical ${ }^{10-12}$, VersaClimbing ${ }^{13}$, Nordic'Track cross-country skiing ${ }^{14}$, in-line skating ${ }^{15}$, rowing ergometry ${ }^{16}$, skiing ergometry ${ }^{17}$, and wheelchair exercise ${ }^{18}$. However, the results for physiological responses to the modes of exercise are varied with some studies finding the alternative mode peak $\mathrm{VO}_{2}$ to be comparable or even higher than treadmill $\mathrm{VO}_{2}{ }^{10,12-14,16}$, while other studies found the treadmill $\mathrm{VO}_{2}$ to be significantly higher than the peak $\mathrm{VO}_{2}$ measured on the other tested modes ${ }^{7-9,15}$. The differences in results are most likely due to muscle mass activation of the exercise mode, familiarity with the exercise, or protocol reliability. Although there seems to be varied results, the previous literature provides additional modes of testing that could be beneficial when alternative means of testing would be preferred when the individual being tested is unable to run or when a more training specific mode of testing is justified.

Despite the myriad research on alternative modalities of $\mathrm{VO}_{2 \max }$ assessments, there are very few published protocols developed for testing maximal aerobic capacity on a Concept2 SkiErg (SE) (Concept2 Inc., Morrisville, VT) ${ }^{17}$. The Concept2 SkiErg has become increasingly popular in gyms, rehabilitation centers, and laboratories due to the small square footage required and total body workout provided. Development of a graded exercise testing protocol for assessing maximal oxygen uptake on a Concept2 SkiErg provides a means of testing populations that are limited in available protocols, including paraplegic athletes, individuals limited to low impact modes of exercise, sport specific modes of athletic testing, and injury recovery requiring a low impact training modality. Protocol development for the SkiErg would alleviate barriers for exercise testing within these paradigms, as well as provide an alternative mode of fitness testing for other laboratories and fitness centers. Prior studies utilizing the SkiErg assessed able-bodied and paraplegic cross-country skiers on a SkiErg and arm ergometer ${ }^{17}$. However, no studies to date have assessed the peak aerobic capacity of averagely fit individuals on a SkiErg compared to a treadmill. Therefore, the purpose of this study was to compare peak aerobic capacity values measured on a treadmill and a Concept2 SkiErg during a graded exercise test (GXT) in no less than averagely fit females. The second purpose was to compare the peak value of heart rate, respiratory exchange ratio, ventilation, and rating of perceived exertion during both GXT on the treadmill and SkiErg.

\section{Methods}

Participants

In total, twenty-two college-aged females completed the current investigation. Descriptive data and anthropometrics are listed in Table 1. All subjects were considered to be averagely fit by American College of Sports Medicine (ACSM) standards, which requires at least 2 days of total body resistance training and a minimum of 3 days of 20 minutes of vigorous cardiovascular exercise or 5 days of 30 minutes of moderate cardiovascular exercise ${ }^{1}$. Recruited participants were excluded if they had a current musculoskeletal injury. Participants completed a Physical Activity Readiness Questionnaire (PAR-Q) to ensure the subject was healthy enough to participate. If any question was answered positive, the subject was excluded from the study. The study was approved by the Liberty University Institutional Review Board, was in accordance with the most recent revisions to the Declaration of Helsinki, and all participants were informed of the benefits and risks of the investigation before completing an institutionally approved informed consent.

Table 1 - Descriptive and anthropometric data.

\begin{tabular}{llll}
\hline Height $\mathbf{( c m})$ & Weight $\mathbf{( k g )}$ & Age $(\mathbf{y r s})$ & Body fat (\%) \\
\hline $165.39 \pm 6.50$ & $64.59 \pm 7.01$ & $21.41 \pm 1.74$ & $24.56 \pm 6.17$ \\
\hline
\end{tabular}

Data presented as mean \pm SD with $n=22$.

Subjects were instructed to avoid strenuous exercise 24 hours before the scheduled test time. Participants were encouraged to get adequate rest the night before as to come into the laboratory well rested. Finally, subjects were asked to eat and hydrate as they would for a hard workout and to come dressed for strenuous exercise. Each participant was scheduled for two separate testing sessions that occurred 72-hours apart. Testing times were kept identical between sessions and consistent to when the individual subject was accustomed to exercising to account for any differences due to circadian rhythm.

Protocol

Upon arriving to the laboratory for both visits, subjects' height, weight, and body composition were measured using a mechanical measuring rod (Seca 216, Seca GmbH, Germany) and bio-electric impedance analysis (InBody 770, InBody, South Korea). 
The two protocols were completed in a counterbalanced order. For both protocols, subjects were fitted with a heart rate sensor on a chest strap (H1 Heart Rate Sensor and Polar Pro Strap, Polar Electro, Finland). Subjects were also fitted with a two-way valve face mask and headpiece (7450 Series Silicone V2 Oro-Nasal Mask, Hans Rudolph, Inc, Shawnee, KS) for breathing analysis. During both testing sessions, a Parvo Medics metabolic cart (TrueOne 2400, Parvo Medics, Sandy, UT) was used for gas analysis. Subjects were briefed on the protocol being done and instructed on proper technique and safety procedures.

To assess the comparison baseline $\mathrm{VO}_{2 \max }$ of the subjects, an accepted incrementally graded treadmill protocol was utilized. Because the subjects were considered at least averagely fit, the Costill/Fox protocol was chosen because it has been shown to elicit higher values in trained individuals than other protocols with different increases in intensity ${ }^{19,20}$. This protocol includes moderate increases in intensity by incrementally elevating the speed and incline. After being fitted with all necessary equipment and calibrating the metabolic cart, subjects began the warm-up on the treadmill. The speed of the treadmill began at 1.0 meters per second $(\mathrm{m} / \mathrm{s})$ and increased by about $0.5 \mathrm{~m} / \mathrm{s}$ each minute until a speed of $3.0 \mathrm{~m} / \mathrm{s}$ was reached. At this point, the speed continued at $3.0 \mathrm{~m} / \mathrm{s}$ for the remainder of the test. The treadmill grade remained at $0 \%$ incline from stage one until the second minute of the speed being set to $3.0 \mathrm{~m} / \mathrm{s}$. On the second minute of $3.0 \mathrm{~m} / \mathrm{s}$, the grade increased to $1 \%$ and continued to increase by $1 \%$ each minute for the duration of the test $^{20}$. The subject was instructed to run until reaching volitional exhaustion. After reaching exhaustion, the subject was instructed to walk on the treadmill, set at $0 \%$ grade and $2.0 \mathrm{~m} / \mathrm{s}$, to recover. Throughout the duration of the test, the subjects' heart rate (HR), respiratory exchange ratio (RER), $\mathrm{VO}_{2}$, ventilation (VE), and rating of perceived exertion (RPE) were recorded each minute. Time to exhaustion (TTE) was also recorded. RPE was measured using the Borg scale with the possible responses being from 6 to $20^{21}$.

The current protocol utilizing the SkiErg was developed and adapted from currently used and published rowing ergometer and skiing ergometer protocols ${ }^{17}$. The intensity of the test was regulated by Watts (W) displayed by the ergometer, while the damper setting remained on level 3 for the entirety of the test. The damper setting controls the amount of air allowed into the flywheel housing and ranges from one being the least air allowed to ten being the most air allowed, thus a damper setting of 3 would allow for less air into the housing and makes it easier to spin the flywheel. After all obligatory equipment was ready and the metabolic cart was prepared, the subject began the warm-up of pulling at 20-30 W for two minutes. After completion of the warm-up, the subject increased intensity to $40-50 \mathrm{~W}$ for the next minute. Increasing the $\mathrm{W}$ was done by either pulling at a faster rate, pulling more forcefully, or a combination of both. The subject continued by increasing the Watt range by $20 \mathrm{~W}$ every minute until reaching volitional exhaustion. A range of $\pm 10 \mathrm{~W}$ outside of the designated intensity range was allowed. Similar to the treadmill, $\mathrm{HR}, \mathrm{VE}, \mathrm{VO}_{2}$, and RPE using the Borg scale were recorded every minute and TTE was recorded following the test.

In order for a participant's test to be considered maximal effort, the criteria outlined by the ACSM guidelines was used $^{22}$. Subjects must have reached at least one of the following: 1) a plateau in $\mathrm{VO}_{2}$ despite an increase in workload, 2) failure of the HR to increase despite increases in workload, 3) an RPE of 17 or greater on the 6-20 RPE scale, 4) peak RER measured at 1.10 or above. Lactate concentration was not used as a criterion for maximal effort due to lactate not being measured in the current study. All subjects reached at least one of these criterions in each test.

\section{Statistical Analysis}

Data were analyzed using SPSS Software (IBM Corporation, Armonk, NY). Descriptive statistics of all subjects were evaluated and the mean and standard deviation (mean \pm SD) were determined for age, height, weight, and body fat percentage. A paired samples t-test was used to determine differences between peak measured values of $\mathrm{HR}, \mathrm{VE}, \mathrm{VO}$, TTE and RER in the treadmill and SkiErg tests. The peak RPE of both tests was compared using a Wilcoxon Signed Rank Test. For all data analysis, the significance level was set at an alpha level of $\mathrm{p} \leq 0.05$.

\section{Results}

There were significant differences between treadmill and SkiErg VO $\mathrm{VO}_{2}, \mathrm{HR}, \mathrm{RER}, \mathrm{VE}, \mathrm{TTE}$, and RPE. Figure 1 presents the means and standard deviations. RER was significantly higher in SkiErg trials than treadmill $(p=0.024)$. In opposition, treadmill measurements for $\mathrm{VO}_{2}$ peak were greater than SkiErg values $(p<0.001)$. HRmax during treadmill exercise was significantly greater than SkiErg maximal heart rates $(p<0.001)$. Similarly, ventilation during treadmill exercise was significantly greater than SkiErg exercise $(p=0.015)$. Time to exhaustion $(p<0.001)$ and ratings of perceived exertion $(p=0.002)$ were both greater during treadmill exercise than SkiErg exercise. 

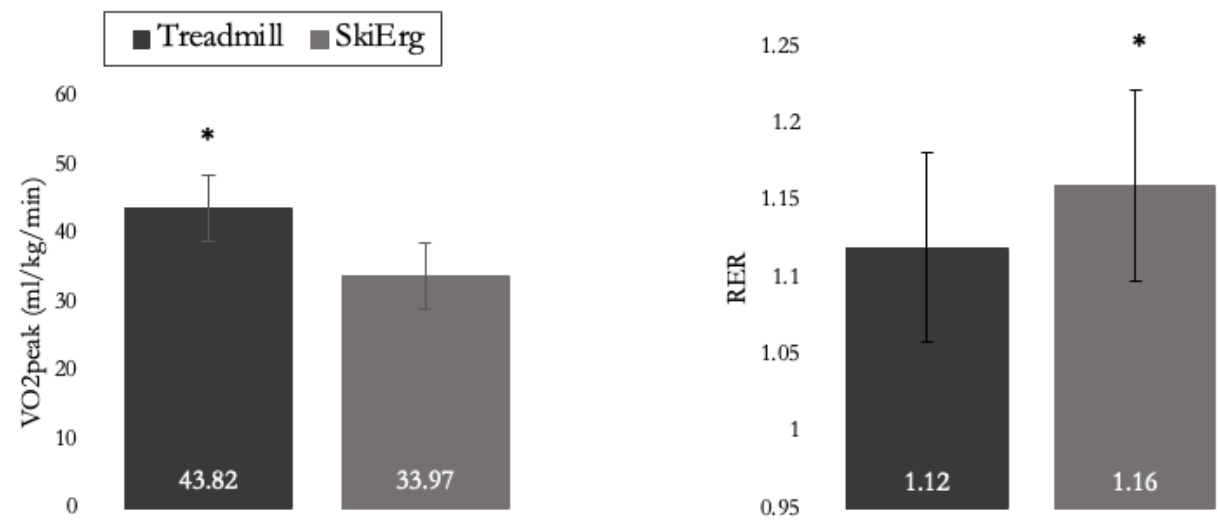

A

B
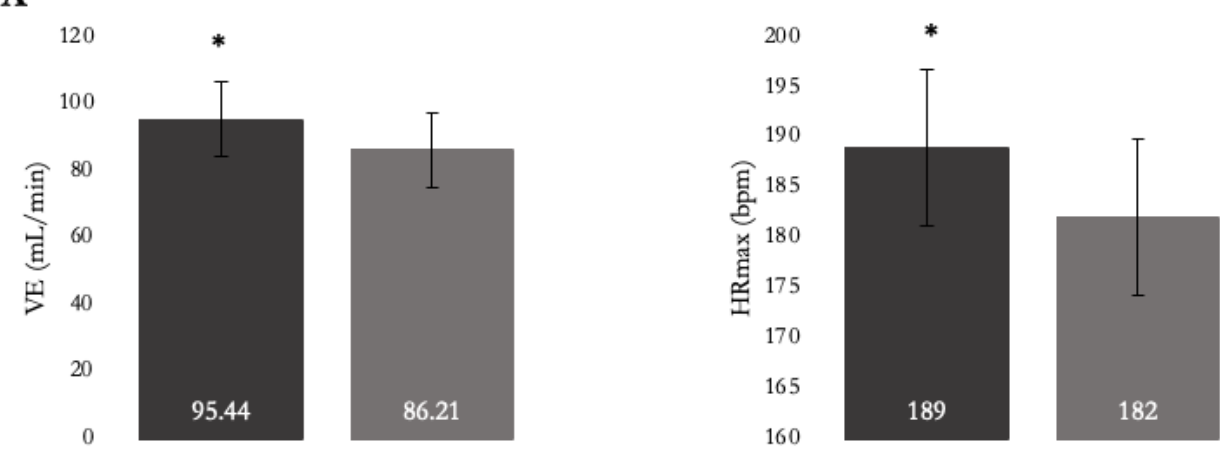

$\mathbf{C}$
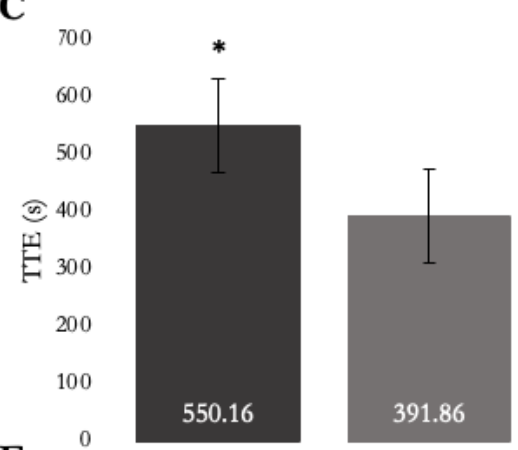

D

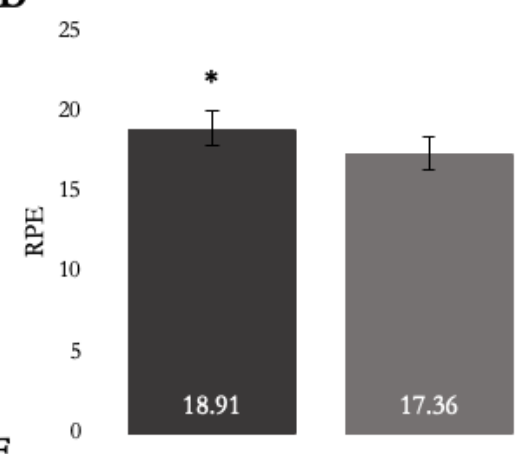

Figure 1 - Comparison of impact of treadmill and SkiErg exercise on (A) $\mathrm{VO}_{2}$ peak = peak oxygen consumption, (B) $\mathrm{RER}=$ respiratory exchange ratio, $(\mathrm{C}) \mathrm{VE}=$ ventilation, $(\mathrm{D}) \mathrm{HR}_{\max }=$ maximal heart rate, $(\mathrm{E}) \mathrm{TTE}=$ time to exhaustion, and $(\mathrm{F}) \mathrm{RPE}=$ ratings of perceived exertion. $*$ Indicates significant difference $(\mathrm{p} \leq 0.05)$

\section{Discussion}

The purpose of this study was to compare peak $\mathrm{VO}_{2}$ and maximum HR, RER, VE, RPE, and TTE on a TM and SE during a GXT protocol in averagely fit college-aged females. From the comparison, it was determined that, in the current population, SE elicited lower values for $\mathrm{VO}_{2}, \mathrm{HR}, \mathrm{RPE}, \mathrm{VE}$, and TTE than the TM. However, SE produced a higher value for RER than the TM (Figure 1).

In the present study, the $\mathrm{VO}_{2}$ max elicited from the treadmill GXT was significantly higher than the $\mathrm{VO}_{2}$ peak measured on the SkiErg GXT (Figure 1). This finding is similar to prior studies that have compared an alternative mode of aerobic exercise to the treadmill for measuring maximal aerobic capacity. Bosak et al. found significantly higher $\mathrm{VO}_{2}$ values on the treadmill when compared to repetitive jumping on a Digi-Jump machine9. Similarly, Wallick et al. and Schrieks et al. elicited higher $\mathrm{VO}_{2}$ values on a treadmill GXT than inline skating and arm crank ergometry, 
respectively ${ }^{8,15}$. The lower measured $\mathrm{VO}_{2}$ values on alternative modes of aerobic testing can be caused by many reasons such as protocol design, population, and physiological responses.

An increase in muscle activation during exercise should yield an increased $\mathrm{VO}_{2 \max }$ during a GXT. With greater muscle activation, there is a greater demand for oxygen at the active tissues. In turn, this increased oxygen demand produces a subsequent increase in oxygen consumption to meet the needs of the muscles being recruited for exercise. Therefore, exercise that activates both the upper and lower body muscles could be predicted to require greater oxygen consumption than exercise of only the lower body ${ }^{25}$.

However, despite the conclusion that greater muscle mass should consistently elicit greater $\mathrm{VO}_{2}$ values, other studies have concluded that there is a range of upper body power output that is ideal for producing higher $\mathrm{VO}_{2}$ measurements. Prior studies have determined that an arm work range of $10-30 \%$ of total work output will increase $\mathrm{VO}_{2}$ peak. However, any arm work higher or lower than this range could potentially negatively impact $\mathrm{VO}_{2}$ peak measurements. This negative result could be due to a higher arm work output than $30 \%$ being supramaximal intensity while the lower body would be contributing submaximal work. The highest $\mathrm{VO}_{2}$ values are elicited when both upper and lower body are performing maximal work ${ }^{23}$. Because total or regional power output was not measured in the present study, the upper body power output cannot be confirmed as a factor in the lower $\mathrm{VO}_{2}$ peak values elicited from the SE. However, this factor should be considered and accounted for in future studies.

Another consideration when testing maximal aerobic capacity is the subjects' training history. Haug et al. have determined that if the population being tested is not upper body trained, a mode of testing using both arms and legs may produce a lower $\mathrm{VO}_{2}$ peak than a leg dominant mode, such as the treadmill ${ }^{14}$. Averagely fit college-aged females are not typically considered to be upper body trained, suggesting that training history could be a factor in producing lower $\mathrm{VO}_{2}$ peak values on the SE than the TM in the present study. Similarly, using a sport specific movement for aerobic testing provides a significant increase in $\mathrm{VO}_{2}$ peak due to movement familiarity. Most participants had no experience with this movement and there were no familiarity days provided to subjects, thus suggesting that unfamiliarity and discomfort with the movement and technique could have been a limiting factor in eliciting higher $\mathrm{VO}_{2}$ values on the $\mathrm{SE}$ in the present study.

The RER in the present study was significantly higher in the SE than the TM (Figure 1). Schrieks et al. suggest that this may be commonly found in modes of exercise and testing with a high upper body work requirement due to lactate production $^{8}$. Due to the relatively smaller muscle mass in the upper body, greater upper body work could increase the lactate production and lead to greater localized muscle fatigue in the arms. This, in turn, could potentially increase the metabolic acidosis and increase the RER higher than leg work alone ${ }^{14}$.

In the present study, VE was found to be significantly lower in the SE than the TM (Figure 1). This is in agreement with Bosak et al. that found lower VE on the TM than Digi-Jump machine, but are conflicting with Haug et al. in their study on the Nordic'Track Ski Simulator ${ }^{9,14}$. Haug et al. suggest that the higher VE on the Nordic'Track was due to the addition of arm work, which has previously shown to increase ventilation ${ }^{14}$. The contradicting results seen in the present study could be due to peripheral fatigue causing early test termination before maximal ventilation was reached. Early termination due to peripheral fatigue in the upper body could also contribute to the findings of maximal HR being significantly greater in the TM than SE (Figure 1). This lower value could be caused by the subjects reaching muscular fatigue prior to reaching ventilatory and cardiopulmonary fatigue.

Both the time to exhaustion and perceived exertion were lower at maximal exertion in the SE than the TM (Figure 1). These findings are similar to those resulting from arm ergometry in females in the study by Schrieks et al. ${ }^{8}$. In this study, it is theorized that the reasoning for shorter TTE was due to peripheral fatigue in the upper body. Additionally, it could be hypothesized that perhaps the protocol utilized in the present study was too aggressive for averagely fit females with no experience with the SkiErg. Because of this fatigue, it could also be hypothesized that lower RPE values are a result of lower overall perceived exertion due to not reaching a true maximal exertion.

\section{Media-Friendly Summary}

Despite there being significant differences in the physiological values between the treadmill and SkiErg, the SkiErg provides sufficient aerobic exercise for many populations. In the present study, the aerobic capacity measured on the SkiErg was $25.3 \%$ lower than that measured on the TM. This percent difference is similar to that of arm ergometry and cycle ergometry which have previously been accepted as alternative modes of aerobic capacity testing ${ }^{14}$. Based on 
this, the Concept2 SkiErg and the current protocol could be used as an alternative mode of testing in averagely fit females, despite not directly comparing to TM GXT maximum values.

\section{Acknowledgements}

The authors thank the research team and the participants involved in the conduction of the experiment. The authors have no conflict of interests to disclose.

\section{References}

1. Dumke C. Health-Related Physical Fitness Testing and Interpretation. In: Dumke C, ed. ACSM's Guidelines for Exercise Testing and Prescription. 10th ed. Philiadelphia, PA: Lippincott Williams and Wilkins; 2018:66-105.

2. Levine BD. V $\square$ O2: What do we know, and what do we still need to know? J Physiol. 2008;586(1):25-34. doi:10.1113/iphysiol.2007.147629

3. Astrand P-O. Experimental Studies of Physical Working Capacity in Relation to Sex and Age. Copenhagen: Ejnar Munksgaard; 1952.

4. Astrand P, Ryhming I. A nomogram for calculation of aerobic capacity (physical fitness) from pulse rate during submaximal work. J Appl Physiol. 1954;7(2):218-221.

5. Londeree BR, Thomas TR, Ziogas G, Smith TD, Zhang Q. \%VO2max versus \%HRmax regressions for six modes of exercise. Med Sci Sports Exerc. 1995;27(3):458-461. doi:10.1249/00005768-199503000-00025

6. Myers J, Buchanan N, Walsh D, et al. Comparison of the ramp versus standard exercise protocols. J Am Coll Cardiol. 1991;17(6):1334-1342. doi:10.1016/S0735-1097(10)80144-5

7. Bar Or O, Zwiren LD. Maximal oxygen consumption test during arm exercise: reliability and validity. J Appl Physiol. 1975;38(3):424-426. doi:10.1152/jappl.1975.38.3.424

8. Schrieks IC, Barnes MJ, Hodges LD. Comparison study of treadmill versus arm ergometry. Clin Physiol Funct Imaging. 2011;31(4):326-331. doi:10.1111/j.1475-097X.2011.01014.x

9. Bosak A, Carnes J, Disponett C, et al. Maximal aerobic capacity comparison between treadmill and repetitive jumping Digi-Jump machine exercise. Med Sci Sport Exerc. 2007;39(5):S346.

10. Dalleck LC, Kravitz L, Robergs RA. Maximal exercise testing using the elliptical cross-trainer and treadmill. J Exerc Physiol Online. 2004;7(3):94-101.

11. Green JM, Crews TR, Pritchett RC, Chaye M, Hall L. Heart rate and ratings of perceived exertion during treadmill and elliptical exercise training. Percept Mot Skills. 2004;98:340-348.

12. Brown AB, Kueffner TE, O’Mahony EC, Lockard MM. Validity of arm-leg elliptical ergometer for VO2max analysis. J Strength Cond Res. 2015;29(6):1551-1555.

13. Brahler CJ, Blank SE. VersaClimbing elicits higher VO2max than does treadmill running or rowing ergometry. Med Sci Sports Exerc. 1995;27(2):249-254. doi:10.1249/00005768-199502000-00015

14. Haug RC, Porcari JP, Brice G, Terry L. Development of a maximal testing protocol for the NordicTrack crosscountry ski simulator. Med Sci Sports Exerc. 1999;31(4):619-623. doi:10.1097/00005768-199904000-00021

15. Wallick ME, Porcari JP, Wallick SB, Berg KM, Brice GA, Arimond GR. Physiological responses to in-line skating compared to treadmill running. Med Sci Sports Exerc. 1995;27(2):242-248.

16. Yoshiga CC, Higuchi M. Heart rate is lower during ergometer rowing than during treadmill running. Eur J Appl Physiol. 2002;87(2):97-100. doi:10.1007/s00421-002-0599-z

17. Baumgart JK, Gürtler L, Gertjan Ettema ·, Sandbakk · Øyvind. Comparison of peak oxygen uptake and exercise efficiency between upper-body poling and arm crank ergometry in trained paraplegic and able-bodied participants. Eur J Appl Physiol. 2018;118(3):1857-1867. doi:10.1007/s00421-018-3912-1

18. Veeger HEJ, Yahmed MH, Van Der Woude LHV, Charpentier P. Peak oxygen uptake and maximal power output of olympic wheelchair-dependent athletes. Med Sci Sports Exerc. 1991;23(10):1201-1209. doi:10.1249/00005768-199110000-00015

19. Kang J, Chaloupka EC, Mastrangelo MA, Biren GB, Robertson RJ. Physiological comparisons among three maximal treadmill exercise protocols in trained and untrained individuals. Eur J Appl Physiol. 2001;84(4):291295. doi:10.1007/s004210000366

20. Costill DL, Fox EL. Energetics of marathon running. Med Sci Sports. 1969;1(2):81-86.

21. Borg GA V. Psychophysical bases of perceived exertion. Med Sci Sports Exerc. 1982;14(5):377-381.

22. Brawner C, Ehrman J, eds. Clinical Exercise Testing and Interpretation. In: ACSM's Guidelines for Exercise Testing and Prescription. 10th ed. Philiadelphia, PA: Lippincott Williams and Wilkins; 2018:111-140.

23. Gleser M, Horstman D, Mello R. The effect on VO2max of adding arm work to maximal leg work. Med Sci Sports. 1974;6(2):104-107. 